\title{
Sleep deprivation impairs recall of social transmission of food preference in rats
}

\author{
This article was published in the following Dove Press journal: \\ Nature and Science of Sleep \\ 4 November 2014 \\ Number of times this article has been viewed
}

\author{
Jessica I Wooden ${ }^{1,2}$ \\ Jennifer Pido' \\ Hunter Mathews' \\ Ryan Kieltyka' \\ Bertha A Montemayor ${ }^{1}$ \\ Christopher P Ward ${ }^{1,3}$ \\ 'Department of Psychology, University \\ of Houston-Clear Lake, ${ }^{2}$ Department \\ of Psychology, University of Houston, \\ ${ }^{3}$ Menninger Department of Psychiatry \\ and Behavioral Sciences, Baylor \\ College of Medicine, Houston, \\ TX, USA
}

Correspondence: Christopher P Ward University of Houston-Clear Lake, Department of Psychology, 2700 Bay Area Blvd, Box 22, Houston, TX 77058, USA

Tel + I 28I 2833303

Emailwardchris@uhcl.edu

\begin{abstract}
Evidence indicates that sleep plays an important role in learning and memory, and disruption of sleep especially seems to interfere with hippocampal memory processes. Social transmission of food preference (STFP), a natural test of paired associative learning, has been shown to be dependent on the hippocampus. While social transmission of food preference is not a novel task, it has not been used to examine the role of sleep in memory consolidation. Male Sprague-Dawley rats were randomly divided into three groups: cage control; sleep-deprived; and device control. Demonstrator rats were given powdered food mixed with a target spice. Test rats then interacted with demonstrator rats before being given a two choice test of powered food with the target spice or a novel spice. Sleep-deprived rats were then placed in an automated device that prevented sleep for 24 hours. After sleep deprivation, animals were given a preference test again to determine memory for the target spice at both 24 hours and 72 hours. Polysomnography was used to validate the method of sleep deprivation. During immediate preference testing, rats demonstrated a clear preference for the food containing the target spice. Rats that experienced 24 hours of sleep deprivation following the initial testing indicated a significant reduction in the recall of the target spice at 24 and 72 hours. The cage control and device animals maintained their preference for food containing the target spice. Therefore, the loss of sleep interfered with memory consolidation for food preference learned via social transmission.
\end{abstract}

Keywords: hippocampus, learning, consolidation

\section{Introduction}

Long-term sleep deprivation has been associated with an increase in health risks, such as hypertension, diabetes, stroke, obesity, and heart attack. ${ }^{1}$ Aside from the possible deleterious health consequences due to sleep deprivation, sleep loss has been shown to impair cognition. Both rodent and human studies have demonstrated that there are a wide variety of cognitive deficits that follow periods of sleep loss, including poorer performance on attention, emotional reactivity, and decision-making tasks. ${ }^{2}$

Sleep appears to play a critical role in the consolidation of certain types of memories, ${ }^{3}$ even though the mechanisms are not fully understood. ${ }^{4}$ As naturally follows, sleep deprivation negatively impacts memory consolidation. ${ }^{5}$ Hippocampal-dependent memory processes are especially susceptible to disruptions of sleep. ${ }^{6}$

Sleep deprivation has been shown to impair the learning of a hippocampaldependent contextual fear task but not an amygdala-dependent cued fear task. ${ }^{7}$ Sleep deprivation impaired rodent performance in the hippocampal-dependent water maze task. ${ }^{8}$ Sleep disruption impacted the learning of a spatial reference memory task, but not of spatial working memory, in the water maze. ${ }^{9}$ Sleep enhanced performance in 
a hippocampal-dependent object-place recognition task, ${ }^{10}$ while sleep deprivation impaired performance in the objectplace recognition task. ${ }^{11}$ Additionally, deficits in hippocampal long-term potentiation have been noted following the disruption of sleep. ${ }^{7,11-13}$

In humans, functional imaging studies have shown that sleep-deprived participants show decreased hippocampal activity during episodic memory learning. ${ }^{14}$ Even mild disruptions of sleep impair hippocampal activation and memory performance. ${ }^{15}$ Patients with chronic insomnia show decreased hippocampal volume. ${ }^{16}$ Sleep also appears to facilitate the consolidation of spatial memory. Participants navigating a virtual world show enhanced recall performance following a night of sleep that is not observed without sleep. ${ }^{17}$

The social transmission of food preference (STFP) task is a natural test of paired associative learning ability that takes advantage of the innate ability of rats to employ olfactory cues to avoid poisoned food. ${ }^{18,19}$ In this task, "observer" rats interact with a "demonstrator" rat that has consumed flavored rat chow. The "observer" rats then show a preference for the target food when given a choice between the flavored chow and a novel alternative. Hippocampal-lesioned observer rats perform similar to control observer rats on the immediate test, but the transfer of food preference is notably decreased when tested 24 hours later. ${ }^{18}$ STFP performance also depends on an intact cholinergic basal forebrain. ${ }^{19,20}$ Unlike other popular tests of hippocampal-dependent memories, such as the water maze task, the STFP does not depend on spatial cues $^{19}$ and does not place rats into a stressful environment. Since sleep loss impacts the performance of memory tasks that depend on the hippocampus, ${ }^{7-9}$ it is hypothesized that the hippocampal-dependent STFP task also depends on sleep for consolidation.

In the present experiment, the role of sleep in rodent performance on the STFP task was tested. Specifically, after learning the STFP task, rats were sleep-deprived before they received a recall test for food preference. It was expected that the loss of sleep would interfere with consolidation and that recall for food preference would be decreased compared with controls that were allowed to sleep. To the authors' knowledge, this was the first test of the role of sleep on STFP task recall.

\section{Methods}

\section{Animals}

Young adult male Sprague-Dawley rats (48-83 days old) (Harlan Laboratories, Inc., Indianapolis, IN, USA) were used in experiments $(\mathrm{N}=32)$. Rats were group-housed in standard cages except during experiments. Rats with surgical implants were individually housed, as were all rats during the 24 hours between the first two behavioral tests. Housing was in a climate controlled facility (room temperature $21^{\circ} \mathrm{C} \pm 1^{\circ} \mathrm{C}$ ), with a 12:12 light-dark cycle (lights on at $10 \mathrm{am}$ ). All behavioral testing was conducted at the beginning of the "lights on" period. Food and water were available ad libitum with the exception of during the behavioral task. All animals were treated in accordance with the Guide for the Care and Use of Laboratory Animals, 8th ed. All procedures were approved by the Institutional Animal Care and Use Committee of the University of Houston-Clear Lake.

\section{Sleep deprivation device}

Rats were sleep-deprived with an automated device (Pinnacle Technology, Lawrence, KS, USA). This device has previously been used in multiple laboratories. ${ }^{21-24}$ The device consists of a cylindrical acrylic cage (30.5 cm in diameter) with a slowly rotating bar (approximately $5 \mathrm{rpm}$ ). The movement of the bar is controlled by an attached computer. The rotating bar "nudges" the rat to prevent sleep. Food and water were available ad libitum in the device. For rats in the behavioral test, food was restricted.

\section{Experimental groups}

Three different experimental groups were utilized during this experiment. Sleep-deprived (SD) rats were individually placed in the sleep-deprivation device for 24 hours. The bar was programmed to spin for 4 second and be off for 12 seconds, repeating for the 24-hour period. Rats in the device control (DC) group were individually placed in the same sleep deprivation device, but the bar was programed to spin for 15 minutes and be off for 45 minutes, repeating for 24 hours. Rats in the cage control (CC) group were individually placed in a similar container with the same bedding, food, and water access.

\section{Polysomnogram recording}

While the commercial sleep deprivation device has been used previously in multiple laboratories, ${ }^{21-24}$ a brief polysomnographic study was conducted to validate this methodology in our laboratory. Male Sprague-Dawley rats $(n=4)$ were used to measure sleep during sleep deprivation. Surgery was carried out under inhalation anesthesia (isoflurane, 1\%-3\%). The rats were also given the analgesic buprenorphine $(0.05$ $\mathrm{mg} / \mathrm{kg}$, subcutaneously) prior to surgery. Bilateral screw electrodes (Plastics One Inc., Roanoke, VA, USA) were fixed onto the skull above the temporal cortex (approximately 
$2 \mathrm{~mm}$ caudal to the bregma and $4 \mathrm{~mm}$ lateral to the midsagittal suture) for electroencephalogram (EEG) recording. Electromyogram (EMG) electrodes consisted of flexible stainless steel wires insulated with nylon, except for approximately $2 \mathrm{~mm}$ of wire, which was stripped of insulation at the end (Plastics One Inc.). EMG wires were inserted into the superior nuchal muscles. After 7 days of recovery from surgery, the rats were placed in the sleep deprivation device and attached to EEG cables (Plastics One, Inc.). Rats received 24 hours of "cable training", in which they habituated to living in the sleep deprivation device, with the electrode cables connected to an overhead swivel. On day 9, the baseline EEGEMG was recorded for 24 hours. On day 10, EEG and EMG activity was recorded during 24 hours of sleep deprivation. The device was turned on at "lights on" and turned off 24 hours later. A Grass Model 15 polygraph with model 15A4 amplifiers (Grass-Telefactor, West Warwick, RI, USA) was used for all EEG and EMG data collection. Behavior was classified into three different states by means of EEG and EMG analysis: awake (Wake), non-REM sleep (NREM), and rapid eye movement sleep (REM). SleepSign software (Kissei Comtech Co, Matsumoto, Japan) was used for off-line EEG and EMG analysis. The recordings were automatically scored in 30-second epochs, followed by visual inspection for accuracy.

\section{Social transmission of food preference}

Separate male Sprague-Dawley rats were randomly assigned to one of three groups: CC ( $n=13)$; $\mathrm{SD}(\mathrm{n}=9)$; and DC $(\mathrm{n}=6)$. Rats were habituated to powdered food 48 hours before the initial experiment. Following this, food was removed 24 hours before the first test. During this time, observer rats were also habituated to the sleep deprivation devices.

On the initial day of testing, demonstrator rats were provided with powdered food (ground Teklad Global 18\% Protein Rodent Diet, Harland Laboratories, Inc.) mixed with the target spice and allowed to eat for 1 hour, from a metal bowl with a lid that sloped toward an access hole to reduce spillage (Lab Products Inc., Seaford, DE, USA). After the hour, the food was weighed to determine that the demonstrators consumed at least 1 gram of the food (a criterion to move to the next step). Observer rats were then presented with a rotation of three demonstrator rats for 20 minutes each, for a total of 1 hour of social interaction. After exposure to demonstrator rats, observer animals were provided with two choices of powdered food in identical bowls, one including the target spice and the other including a novel spice. Rats were given 1 hour to eat, and the amount of food eaten from each jar was weighed and recorded. Observer rats were then placed in either sleep deprivation device (SD or DC) or placed in a similar container (CC) for 24 hours. Food preference was retested at 24 hours following the initial test. All rats were returned to standard housing cages and then retested 72 hours after the initial test. Figure 1 provides a schematic depiction of the experimental design.

The spices used were powdered thyme $(2 \% \mathrm{w} / \mathrm{w})$, basil $(0.7 \% \mathrm{w} / \mathrm{w})$, ginger $(1 \% \mathrm{w} / \mathrm{w})$, and clove $(0.25 \% \mathrm{w} / \mathrm{w})$, mixed into powdered rat chow in the aforementioned concentrations. Spice pairs were either thyme and basil, or ginger and clove. Pilot testing indicated that rats did not show an initial preference to a particular spice when these pairs were presented (data not shown). Presentation order was counterbalanced, and all spices were used as the target spice at one point during the experiment.

\section{Statistics analysis}

All data are reported as mean \pm standard error. Polysomnography data were analyzed by a repeated measures analysis of variance (ANOVA), with hour and day as repeated measures. Percentage of time in each behavioral state (Wake, NREM, and REM) was the main dependent variable. The STFP task data were analyzed by a mixed model ANOVA, with day (baseline, 24-hour recall, and 72-hour recall) as a repeated measure variable and group $(\mathrm{CC}, \mathrm{SD}$, and $\mathrm{DC})$ and as a between-group variable. Post hoc comparisons were made with a Fisher's test. All data analysis was conducted

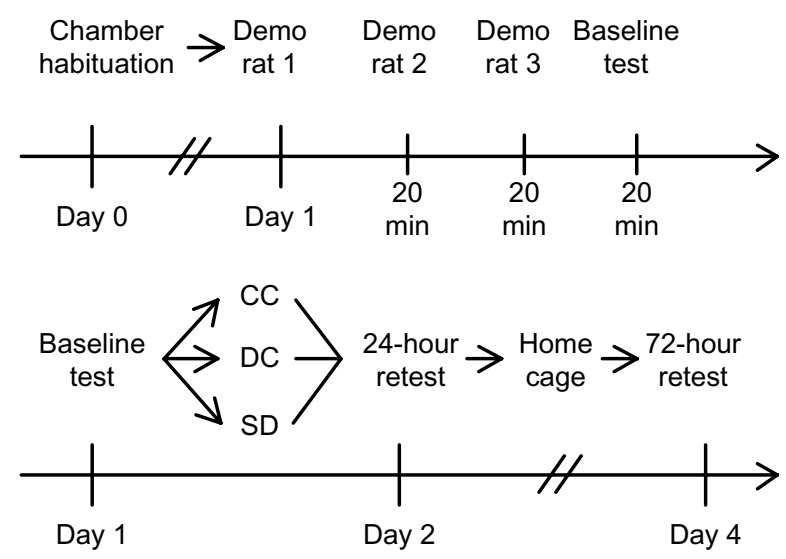

Figure I Schematic figure depicting experimental design.

Notes: Top line represents the initial day of testing. After being habituated to the experimental chambers for 24 hours, rats were exposed to three different demonstrator rats. The rats were then given a baseline preference task. Bottom line represents the testing and manipulation of rats. Following baseline testing, rats were placed in either CC, DC, or SD for 24 hours. Immediately following, rats were preference-tested again (24-hour retest). All rats were returned to their home cage for 2 days before being given a final preference-testing (72-hour retest).

Abbreviations: CC, cage control; DC, device control; Demo, demonstrator; SD, sleep-deprived. 
utilizing SPSS Statistics for Windows, Version 20.0 (IBM Corp, Armonk, NY, USA), with an alpha level of 0.05 .

\section{Results}

\section{Polysomnography}

Figure 2 illustrates the percentage of time animals spent in Wake, NREM, or REM respectively before, during, and after sleep deprivation. For Wake (Figure 2A), there was a significant main effect of day $(F[2,6]=101.73, P<0.001)$,
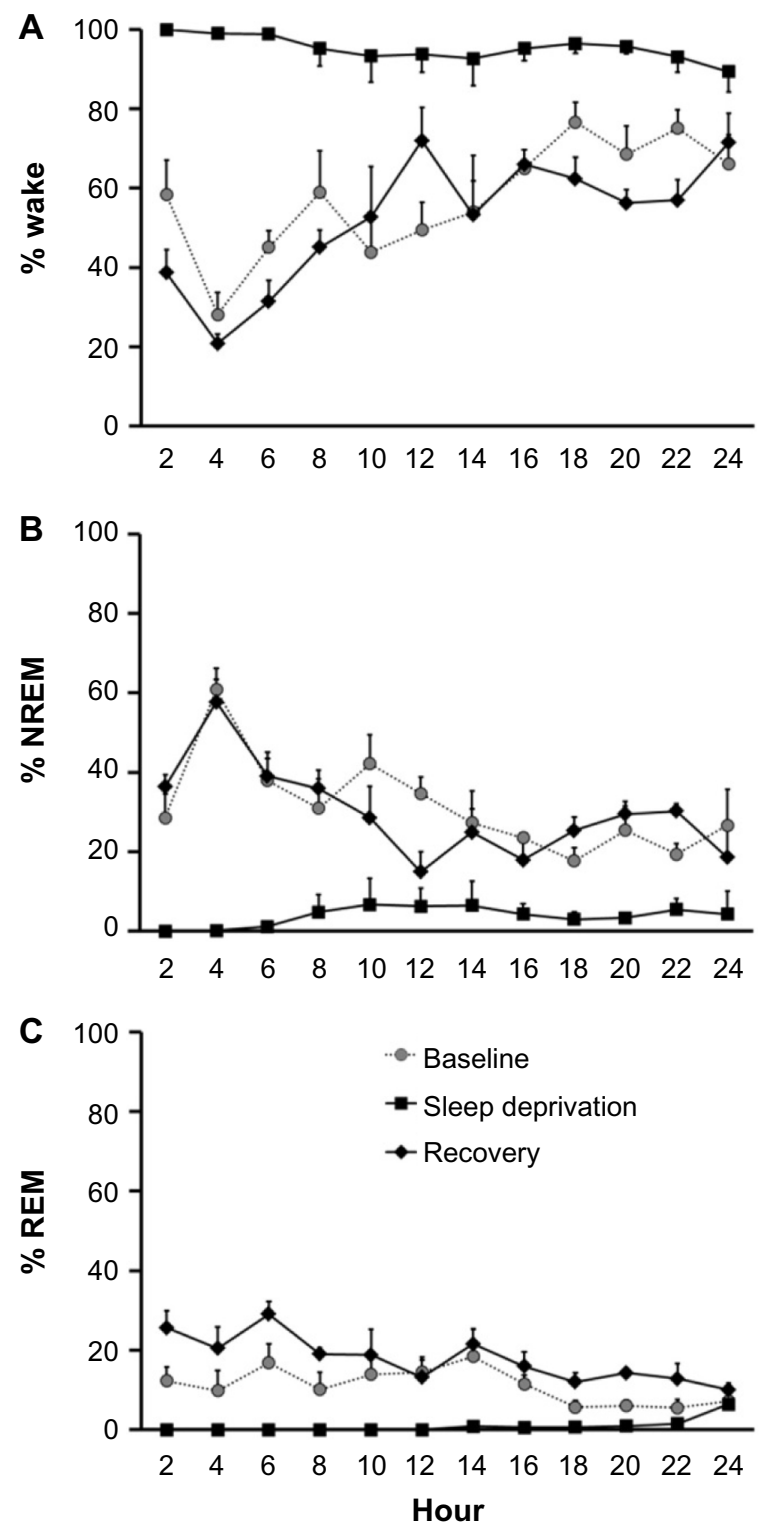

Figure 2 Sleep deprivation protocol reduced amount of time spent in NREM and REM sleep.

Notes: Figure illustrates results of polysomnography of rodents over 3 consecutive days, during baseline (gray dot), sleep deprivation (black square), and recovery (black diamond). Data points represent the mean and SEM percentage of time spent in (A) wake, (B) NREM sleep, and (C) REM sleep. Data points were averaged in 2-hour bins. Lights were on during hours 0-12, and lights were off during hours 12-24.

Abbreviations: NREM, non-REM; REM, rapid eye movement; SEM, standard error of the mean. where sleep-deprived animals spent significantly more time in Wake (95.3\% $\pm 3.2 \%)$ than during baseline $(57.4 \% \pm 2.5 \%)$ $(P=0.005)$ or recovery $(52.2 \% \pm 2.6 \%)(P=0.005)$. There was no significant difference between baseline and recovery $(P=0.305)$. Percentage of time in NREM sleep showed the opposite (Figure 2B). There was a significant main effect $(F[2,6]=75.73, P<0.001)$, indicating that rats during sleep deprivation spent significantly less time in NREM sleep, $4.5 \% \pm 2.9 \%$ as compared with baseline $(34.6 \% \pm 1.0 \%)$ $(P=0.009)$ and recovery $(35.7 \% \pm 3.0 \%)(P=0.001)$. There was no significant difference between baseline and recovery $(P=1.00)$. REM sleep (Figure 2C) also showed a significant main effect $(F[2,6]=26.537, P=0.001)$. Only the amount of REM sleep during recovery $(17.7 \% \pm 1.7 \%)$ was significantly higher than during sleep deprivation $(0.9 \% \pm 0.5 \%)(P=0.014)$. The decrease in REM sleep during sleep deprivation as compared with baseline $(10.1 \% \pm 2.1 \%)$ approached significance $(P=0.051)$. There was no significant difference between baseline and recovery $(P=0.251)$.

\section{Social transmission of food preference}

Figure 3 represents the percentage of target spice that rats consumed during baseline, 24-hour recall, and 72-hour recall testing. There was a significant group $\mathrm{X}$ trial interaction $(F[4,50]=3.562, P=0.012)$. During baseline testing (Figure $3 \mathrm{~A}$ ), there were no significant differences in percentage of target spice consumed between $\mathrm{CC}$ and DC $(P=0.925), \mathrm{DC}$ and SD $(P=0.307)$, or $\mathrm{CC}$ and SD $(P=0.089)$. Following 24 hours of sleep deprivation or device control (Figure 3B), rats were tested for 24-hour recall of the target spice. SD rats consumed a significantly lower percentage of target spice than both $\mathrm{CC}(P=0.002)$ and DC $(P=0.006)$. There was no significant difference between $C C$ and DC $(P=0.914)$. Rats were then returned to their home cages. A preference test was given again at 72 hours after baseline (Figure 3C). As before, SD rats consumed significantly less of the target spice than both $\mathrm{CC}(P=0.001)$ and $\mathrm{DC}(P=0.027)$. There was no significant difference between $\mathrm{CC}$ and DC $(P=0.474)$.

Comparing across the three testing periods, the percentage of target spice consumed by the CC group did not significantly differ from baseline testing at the retest after 24 hours $(P=0.162)$ or 72 hours $(P=0.116)$. The percentage of target spice consumed by DC also did not significantly differ from baseline testing at the retest after 24 hours $(P=0.201)$ or 72 hours $(P=0.863)$; on the other hand, the percentage of target spice consumed by SD did significantly decrease from baseline at 24 hours $(P=0.0495)$ and 72 hours $(P=0.014)$. SD 
A

Baseline

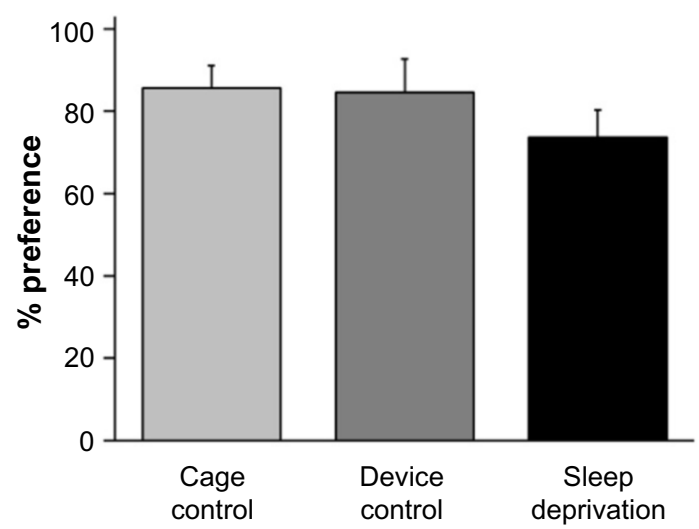

B

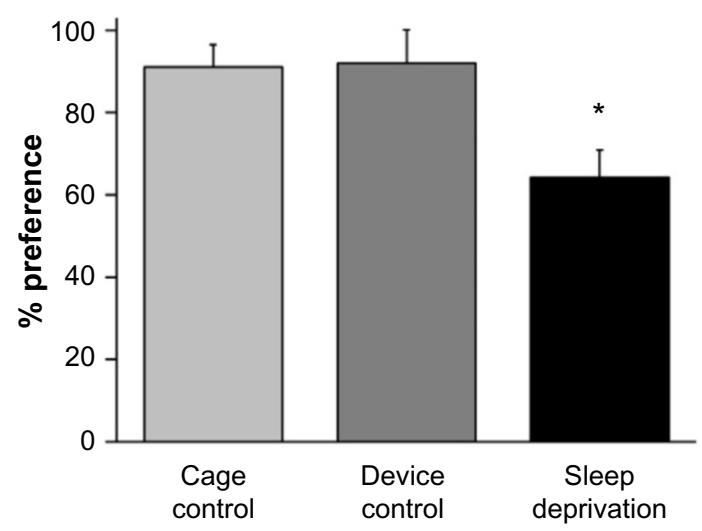

C 72-hour retest

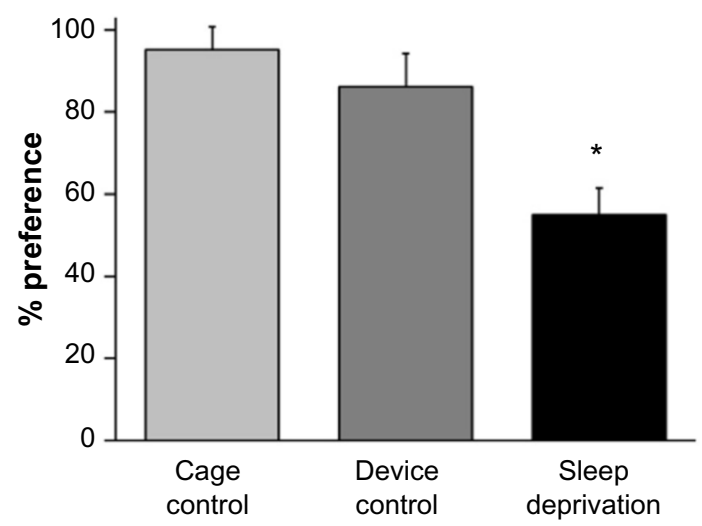

Figure 3 Twenty-four hours of sleep deprivation impaired recall of food preference immediately following sleep deprivation and at later retest.

Notes: Figure shows mean and SEM percentage of total food consumed containing the target spice of the CC $(n=\mid 3), D C(n=6)$, and SD $(n=6)$ groups. (A) Percentage of food preference of observer rats after interacting with demonstrator rats. There were no manipulations among groups, and no significant differences were noted. After baseline testing, rats were moved to either the CC chamber or the sleep deprivation chamber where they experienced either the DC or SD for 24 hours. (B) Percentage of food preference of the target spice following 24 hours of no manipulation (CC), occasional stimulation (DC), or constant stimulation (SD). SD rodents recalled a significantly lower percentage of the target spice than did both the $C C(P=0.002)$ and $D C(P=0.006)$ rodents. $(C)$ Percentage of food preference of the target spice 2 days later. SD rodents again recalled significantly less than did the $C C(P=0.00 I)$ and $D C(P=0.027)$ rodents. $* P<0.05$.

Abbreviations: $C C$, cage control; DC, device control; Demo, demonstrator; $\mathrm{SD}$, sleep-deprived; SEM, standard error of the mean. performance during the retests at 24 hours and 72 hours did not significantly differ $(P=0.133)$.

\section{Discussion}

The present experiment demonstrates that 24 hours of sleep deprivation impairs the recall of a naturalistic paired associative learning task. The STFP is a hippocampal-dependent $\operatorname{task}^{18}$ that does not rely on spatial cues. ${ }^{19}$ These results are similar to other studies demonstrating that sleep deprivation impairs the recall of a hippocampal-dependent task..$^{7-9}$

Sleep deprivation was accomplished through an automated device that produced over $95 \%$ wakefulness in animals across a 24-hour period. Most effectively eliminated was REM sleep, for which a noticeable rebound effect is observed during the first 6 hours of recovery sleep. Even though sample sizes were small, there was a clear change in sleep/wake behavior that produced a meaningful change in the behavioral task. Polysomnography was not performed on DC animals since there was no effect of the control procedure on the behavioral task. During observation of rats in the DC group, behavior was as expected. Rats would sleep when given the opportunity and wake when the rod turned. However, this is a limitation in the study. It is possible that some sleep was lost in the DC group and that some critical amount of sleep is needed to successfully recall the target spice. Without the direct measure of sleep in experimental animals, actual sleep/wake behaviors must be inferred. It should be noted that only male rats were tested in all experiments. Future research should confirm whether findings generalize to female rats as well.

The STFP task is a hippocampal-dependent task, independent of the spatial context. Specifically, the hippocampus is implicated in intermediate retention of memory (1-14 days) in the STFP task. ${ }^{25}$ For longer periods of retention of the STFP task, starting around 3 weeks, the hippocampus seems to become less important. ${ }^{26}$ Performance deficits in the task were noted if hippocampal lesions were made 1 day after training, but not 21 to 30 days later, possibly pointing to a small window for consolidation of hippocampal-based tasks. ${ }^{26}$ Sleep deprivation has been shown to cause a reduction in phosphorylated cyclic adenosine monophosphate (cAMP) response element-binding protein (CREB) in the hippocampus. ${ }^{27}$ Proper functioning of the CREB pathway is necessary for long-term retention (11 days) of the STFP task but not for short-term retention (30 minutes). ${ }^{28}$ Mice with mutated CREB performed normally in 24-hour retention of the STFP. ${ }^{29}$ This independence of CREB, which regulates transcription, suggests that a hippocampal-mediated intermediate-term memory ${ }^{30}$ is being 
used by rats as opposed to long-term memory. ${ }^{31}$ However, aged rats with decreased phosphorylated CREB perform worse than young control rats on recall of the STFP task 48 hours after training but are similar to young control rats during immediateterm and 24-hour testing. ${ }^{32}$ This makes defining the memory systems involved during testing at 72 hours more difficult, though if information was not properly encoded before retesting at 24 hours, it stands to reason that recall will continue to be poor at 72 hours.

The role of sleep in the consolidation of memories also has a critical period. REM sleep deprivation 4 to 8 hours after training has been shown to impair spatial water maze performance. ${ }^{33}$ In a recent study utilizing a hippocampaldependent object-place recognition task, sleep deprivation was found to impair memory and hippocampal long-term potentiation for 1 to 4 hours after training. ${ }^{11}$ The present study demonstrates that the STFP task performance is similarly sensitive to sleep loss following training. Future research can manipulate the timing of sleep deprivation within the initial 24-hour period following training, to determine more precisely where the critical period lies.

To the authors' knowledge, this report is the first demonstration that sleep deprivation impairs the consolidation of memory of a social exchange of food preference in rats. Rats are social creatures and communicate socially about food preferences. Rats will eat food that they smelled on a demonstrator rat, unless the demonstrator rat is dead. ${ }^{34,35}$ This social communication of food preference serves a vital role in the animal's ability to thrive, and the inability to distinguish between what food is viable and what food is not could lead to a lower survival rate. Therefore, the STFP task is an example of a natural form of communication between rodents. This naturalistic task therefore carries more ecological validity than many other tests of hippocampal function, such as water maze learning. Additionally, unlike the water maze task, the STFP task does not rely on placing rodents into a stressful testing environment. The STFP task also does not rely on negative reinforcement, such as the water maze, or negative associations, such as a contextual fear task. The addition of the STFP task to the various other hippocampal-dependent tasks that are impacted by sleep loss will give investigators a new tool to examine sleep's role in the consolidation of memories.

\section{Acknowledgments}

This work was supported by the National Institute of Mental Health (grant number R15MH087934) and a Faculty Research Support Fund awarded to CPW.

\section{Disclosure}

The authors report no conflicts of interest in this work.

\section{References}

1. Colten HR, Altevogt BM, editors. Sleep Disorders and Sleep Deprivation: An Unmet Public Health Problem. Washington, DC: National Academies Press (US); 2006.

2. McCoy JG, Strecker RE. The cognitive cost of sleep lost. Neurobiol Learn Mem. 2011;96(4):564-582.

3. Diekelmann S, Born J. The memory function of sleep. Nat Rev Neurosci. 2010;11(2):114-126.

4. Frank MG, Benington JH. The role of sleep in memory consolidation and brain plasticity: dream or reality? Neuroscientist. 2006;12(6): 477-488.

5. Stickgold R, Walker MP. Sleep-dependent memory consolidation and reconsolidation. Sleep Med. 2007;8(4):331-343.

6. Walker MP. Cognitive consequences of sleep and sleep loss. Sleep Med. 2008;9 Suppl 1:S29-S34.

7. McDermott CM, LaHoste GJ, Chen C, Musto A, Bazan NG, Magee JC. Sleep deprivation causes behavioral, synaptic, and membrane excitability alterations in hippocampal neurons. J Neurosci. 2003;23(29):9687-9695.

8. Guan Z, Peng X, Fang J. Sleep deprivation impairs spatial memory and decreases extracellular signal-regulated kinase phosphorylation in the hippocampus. Brain Res. 2004;1018(1):38-47.

9. Ward CP, McCarley RW, Strecker RE. Experimental sleep fragmentation impairs spatial reference but not working memory in Fischer/Brown Norway rats. J Sleep Res. 2009;18(2):238-244.

10. Binder S, Baier PC, Mölle M, Inostroza M, Born J, Marshall L. Sleep enhances memory consolidation in the hippocampus-dependent objectplace recognition task in rats. Neurobiol Learn Mem. 2012;97(2): 213-219.

11. Prince TM, Wimmer M, Choi J, Havekes R, Aton S, Abel T. Sleep deprivation during a specific 3 -hour time window post-training impairs hippocampal synaptic plasticity and memory. Neurobiol Learn Mem. 2014;109:122-130.

12. Davis CJ, Harding JW, Wright JW. REM sleep deprivation-induced deficits in the latency-to-peak induction and maintenance of long-term potentiation within the CA1 region of the hippocampus. Brain Res. 2003;973(2):293-297.

13. Tartar JL, Ward CP, McKenna JT, et al. Hippocampal synaptic plasticity and spatial learning are impaired in a rat model of sleep fragmentation. Eur J Neurosci. 2006;23(10):2739-2748.

14. Yoo SS, Hu PT, Gujar N, Jolesz FA, Walker MP. A deficit in the ability to form new human memories without sleep. Nat Neurosci. 2007;10(3):385-392.

15. Van Der Werf YD, Altena E, Schoonheim MM, et al. Sleep benefits subsequent hippocampal functioning. Nat Neurosci. 2009;12(2): 122-123.

16. Riemann D, Voderholzer U, Spiegelhalder K, et al. Chronic insomnia and MRI-measured hippocampal volumes: a pilot study. Sleep. 2007;30(8):955-958.

17. Ferrara M, Iaria G, Tempesta D, et al. Sleep to find your way: the role of sleep in the consolidation of memory for navigation in humans. Hippocampus. 2008;18(8):844-851.

18. Bunsey M, Eichenbaum H. Selective damage to the hippocampal region blocks long-term retention of a natural and nonspatial stimulus-stimulus association. Hippocampus. 1995;5(6):546-556.

19. Berger-Sweeney J, Stearns NA, Frick KM, Beard B, Baxter MG. Cholinergic basal forebrain is critical for social transmission of food preferences. Hippocampus. 2000;10(6):729-738.

20. Vale-Martínez A, Baxter MG, Eichenbaum H. Selective lesions of basal forebrain cholinergic neurons produce anterograde and retrograde deficits in a social transmission of food preference task in rats. Eur $J$ Neurosci. 2002;16(6):983-998. 
21. Hines DJ, Schmitt LI, Hines RM, Moss SJ, Haydon PG. Antidepressant effects of sleep deprivation require astrocyte-dependent adenosine mediated signaling. Transl Psychiatry. 2013;3:e212.

22. Wisor JP, Clegern WC, Schmidt MA. Toll-like receptor 4 is a regulator of monocyte and electroencephalographic responses to sleep loss. Sleep. 2011;34(10):1335-1345.

23. Naylor E, Aillon DV, Barrett BS, et al. Lactate as a biomarker for sleep. Sleep. 2012;35(9):1209-1222.

24. Sims RE, Wu HH, Dale N. Sleep-wake sensitive mechanisms of adenosine release in the basal forebrain of rodents: an in vitro study. PLoS One. 2013;8(1):e53814.

25. van der Kooij MA, Sandi C. Social memories in rodents: methods, mechanisms and modulation by stress. Neurosci Biobehav Rev. 2012;36(7):1763-1772.

26. Clark RE, Broadbent NJ, Zola SM, Squire LR. Anterograde amnesia and temporally graded retrograde amnesia for a nonspatial memory task after lesions of hippocampus and subiculum. J Neurosci. 2002;22(11): 4663-4669.

27. Vecsey CG, Baillie GS, Jaganath D, et al. Sleep deprivation impairs cAMP signalling in the hippocampus. Nature. 2009;461(7267):1122-1125.

28. Brightwell JJ, Smith CA, Countryman RA, Neve RL, Colombo PJ. Hippocampal overexpression of mutant creb blocks long-term, but not short-term memory for a socially transmitted food preference. Learn Mem. 2005;12(1):12-17.
29. Gass P, Wolfer DP, Balschun D, et al. Deficits in memory tasks of mice with CREB mutations depend on gene dosage. Learn Mem. 1998;5(4-5):274-288.

30. Sutton MA, Bagnall MW, Sharma SK, Shobe J, Carew TJ. Intermediate-term memory for site-specific sensitization in aplysia is maintained by persistent activation of protein kinase C. J Neurosci. 2004;24(14):3600-3609.

31. Lukowiak K, Adatia N, Krygier D, Syed N. Operant conditioning in Lymnaea: evidence for intermediate- and long-term memory. Learn Mem. 2000;7(3):140-150.

32. Countryman RA, Gold PE. Rapid forgetting of social transmission of food preferences in aged rats: relationship to hippocampal CREB activation. Learn Mem. 2007;14(5):350-358.

33. Smith C, Rose GM. Evidence for a paradoxical sleep window for place learning in the Morris water maze. Physiol Behav. 1996;59(1): 93-97.

34. Galef BG. Direct and indirect behavioral pathways to the social transmission of food avoidance. Ann N Y Acad Sci. 1985;443: 203-215.

35. Galef BG Jr, Wigmore SW. Transfer of information concerning distant foods: a laboratory investigation of the 'information-centre' hypothesis. Anim Behav. 1983;31(3):748-758.
Nature and Science of Sleep

\section{Publish your work in this journal}

Nature and Science of Sleep is an international, peer-reviewed, open access journal covering all aspects of sleep science and sleep medicine, including the neurophysiology and functions of sleep, the genetics of sleep, sleep and society, biological rhythms, dreaming, sleep disorders and therapy, and strategies to optimize healthy sleep. The journal welcomes

\section{Dovepress}

original research, clinical \& epidemiological studies, reviews \& evaluations, case reports and extended reports. The manuscript management system is completely online and includes a very quick and fair peerreview system, which is all easy to use. Visit http://www.dovepress.com/ testimonials.php to read real quotes from published authors. 